\title{
Avaliação dos resultados da artroplastia parcial de ombro para tratamento da artropatia por lesão do manguito rotador
}

\author{
Evaluating the results of partial shoulder arthroplasty \\ for the treatment of arthropathy due to rotator cuff lesion \\ Sérgio luiz Checchia ${ }^{1}$, Pedro Doneux Santos ${ }^{4}$, Alberto Naoki MiyazakI ${ }^{2}$, \\ Marcelo Fregoneze ${ }^{3}$, Luciana Andrade da Silva ${ }^{4}$, Flávio Santos Ferreira Leite ${ }^{5}$, Caio Zamboni ${ }^{5}$
}

\section{RESUMO}

Objetivo: Avaliar os resultados obtidos com a artroplastia parcial de ombro para o tratamento da artropatia por lesão do manguito rotador. Métodos: No período de junho de 1989 a março de 2004, 11 ombros de 11 pacientes foram submetidos a artroplastia parcial de ombro para o tratamento de artropatia por LMR. $O$ tempo de seguimento variou de 27 a 183 meses, com média de 69 meses. A idade

* Trabalho realizado no Departamento de Ortopedia e Traumatologia da Faculdade de Ciências Médicas da Santa Casa de São Paulo (DOT-FCMSC-SP), Pavilhão "Fernandinho Simonsen". Diretor: Prof. Dr. Cláudio Santili - São Paulo (SP), Brasil.

1. Doutor, Professor Adjunto da Faculdade de Ciências Médicas da Santa Casa de São Paulo - FCMSCSP - São Paulo (SP), Brasil.

2. Doutor, Professor Instrutor da Faculdade de Ciências Médicas da Santa Casa de São Paulo - FCMSCSP - São Paulo (SP), Brasil.

3. Professor Assistente da Faculdade de Ciências Médicas da Santa Casa de São Paulo - FCMSCSP - São Paulo (SP), Brasil.

4. Assistente do Grupo de Ombro e Cotovelo do Departamento de Ortopedia e Traumatologia da Faculdade de Ciências Médicas da Santa Casa de São Paulo - FCMSCSP - São Paulo (SP), Brasil.

5. Estagiário do Grupo de Ombro e Cotovelo do Departamento de Ortopedia e Traumatologia da Faculdade de Ciências Médicas da Santa Casa de São Paulo - FCMSCSP - São Paulo (SP), Brasil.

Endereço para correspondência: Rua Dr. Cesário Mota Júnior, 112 - 01220-020 - São Paulo (SP). Tel./fax: (11) 3222-6866.

E-mail: ombro@ombro.med.br

Recebido em 3/3/07. Aprovado para publicação em 28/5/08.

Copyright RBO2008 média foi de 69,1 anos, variando de 44 a 78 anos. Houve predomínio do sexo feminino em $90,9 \%$ dos casos (10 pacientes). $O$ membro dominante foi acometido em 10 pacientes. Os métodos escolhidos para avaliação dos pacientes, no seguimento pós-operatório, basearam-se nos critérios da UCLA; no índice de satisfação dos pacientes em relação ao alívio da dor; e no método de avaliação de objetivos limitados, proposto por Neer et al. Resultados: $O$ índice de satisfação dos pacientes em relação ao alívio da dor foi de 81,8\%. Com o método de avaliação da UCLA, observamos média de 22,7 pontos. Por meio do método de avaliação de objetivos limitados, proposto por Neer $e t$ al, obtivemos seis pacientes com resultados satisfatórios e cinco insatisfatórios. Conclusão: A artroplastia parcial de ombro para o tratamento da artropatia por lesão do manguito rotador promove alívio do quadro doloroso e permite alguma melhora na função do membro para realização das atividades diárias; entretanto, é uma técnica que leva a altos índices de resultados insatisfatórios.

Descritores - Ombro; Artroplastia; Artropatias; Bainha rotadora

\section{ABSTRACT}

Objective: To evaluate the results achieved with partial arthroplasty of the shoulder to treat 
arthropathy due to rotator cuff lesion. Methods: From June 1989 to March 2004, 11 shoulders of 11 patients were submitted to partial arthroplasty of the shoulder to treat an arthropathy due to rotator cuff lesion. Follow-up time varied from 27 to 182 months, with a mean of 69 months. Mean age was 69.1 years, ranging from 44 to 78 years. There was a predominance of the female gender, in $90.9 \%$ of the cases (10 patients). The dominant member was involved in 10 patients. The methods chosen to evaluate the patients in follow-up were based on UCLA criteria; on patient satisfaction indices regarding pain relief; and on limited objective evaluation method proposed by Neer et al. Results: The patient satisfaction index regarding pain relief was $81.8 \%$. With the UCLA evaluation method, the authors observed a mean score of 22.7 points. With the limited objective evaluation method proposed by Neer et al, they had six patients with satisfactory results and five with unsatisfactory results. Conclusion: Partial arthroplasty of the shoulder to treat an arthropathy due to rotator cuff lesion brings pain relief and allows for some improvement in limb function for daily activities, however, the technique has high indices of unsatisfactory results.

Keywords - Shoulder; Arthroplasty; Joint diseases; Rotator cuff

\section{INTRODUÇÃO}

O manguito rotador (MR) possui importante função na estabilização dinâmica do ombro. Atua exercendo forças compressivas, centralizando a cabeça do úmero na cavidade glenoidal. Antagonicamente a essas forças compressivas, existem forças de cisalhamento resultantes da ação muscular do deltóide, que tendem a tracionar a cabeça do úmero para cima ${ }^{(1-2)}$. Dessa forma, uma carga excêntrica é aplicada na cabeça do úmero contra o arco coracoacromial ${ }^{(1-4)}$.

Neer et al descreveram a artropatia por lesão do manguito rotador (LMR) como o produto final de alterações mecânicas e nutricionais da articulação do ombro, condição que ocorre em aproximadamente $4 \%$ dos pacientes com LMR completa ${ }^{(5)}$. Sanchez-Sotelo ob- servaram que alguns pacientes com LMR extensa, de longo tempo de evolução, eventualmente podem evoluir com alterações degenerativas da articulação do ombro $^{(6)}$.

A artroplastia do ombro foi introduzida e popularizada por Neer, sendo realizada desde 1951. No entanto, as primeiras descrições da artroplastia parcial de ombro para tratamento da artropatia por LMR datam de 1982, quando Neer as realizou em 16 pacientes, obtendo resultados satisfatórios em 10, com seguimento mínimo de 24 meses $^{(7)}$. A artroplastia parcial de ombro tem sido realizada com melhores resultados em comparação ao procedimento total, para pacientes com artropatia por LMR, pois a artroplastia total de ombro, quando associada à migração proximal da cabeça do úmero, possui como complicação freqüente a soltura do componente glenoidal $^{(1,8)}$.

O objetivo deste estudo é avaliar os resultados obtidos com a artroplastia parcial de ombro para o tratamento da artropatia por LMR.

\section{MÉTODOS}

No período de junho de 1989 a março de 2004, 11 ombros de 11 pacientes foram submetidos a artroplastia parcial de ombro para o tratamento de artropatia por LMR. As cirurgias foram realizadas pelo Grupo de Cirurgia de Ombro e Cotovelo do Departamento de Ortopedia e Traumatologia da Faculdade de Ciências Médicas da Santa Casa de Misericórdia de São Paulo - Pavilhão "Fernandinho Simonsen".

Foram incluídos nesta série, retrospectiva, todos os pacientes com artropatia por LMR que não melhoraram com tratamento por reabilitação por no mínimo seis meses, que apresentavam à avaliação pré-operatória sinais e sintomas de osteartrose do ombro com lesões irreparáveis do MR e que foram submetidos à artroplastia parcial do ombro como tratamento de escolha. Foram considerados critérios de exclusão pacientes com artropatia por LMR que melhoraram com tratamento clínico, pacientes com lesões extensas, mas reparáveis do MR e pacientes com lesões irreparáveis do MR, mas sem osteartrose do ombro.

A média de idade dos pacientes no momento da cirurgia foi de 69 anos, variando de 44 a 78 anos. Houve 
predomínio do sexo feminino em 10 pacientes $(90,9 \%)$. $\mathrm{O}$ membro dominante era o acometido em 10 pacientes $(90,9 \%)$ (tabela 1$)$.

O tempo decorrido do início da sintomatologia até a realização do procedimento cirúrgico variou de sete a 96 meses, com média de 49 meses. Foi realizado tratamento conservador prévio com fisioterapia para to- dos os pacientes, sendo associado à infiltração local com corticosteróide em cinco casos. Entre os pacientes, dois foram submetidos a procedimento cirúrgico prévio, tendo sido realizada a acromioplastia por via aberta. Um destes pacientes apresentou denervação do músculo deltóide como complicação da acromioplastia por via aberta (tabela 1).

\section{TABELA 1}

Dados dos pacientes operados com artroplastia parcial do ombro devido a artropatia por lesão do manguito rotador quanto à epidemiologia, lesões associadas e resultados

\begin{tabular}{|c|c|c|c|c|c|c|c|c|c|c|c|c|c|c|c|}
\hline $\begin{array}{l}\text { Número } \\
\text { do } \\
\text { paciente }\end{array}$ & $\begin{array}{l}\text { Idade } \\
\text { (anos) }\end{array}$ & Sexo & $\begin{array}{c}\text { Lado } \\
\text { dominante }\end{array}$ & $\begin{array}{l}\text { Cirurgia } \\
\text { prévia }\end{array}$ & $\begin{array}{l}\text { Tempo } \\
\text { até } \\
\text { cirurgia } \\
\text { (meses) }\end{array}$ & $\begin{array}{l}\text { Classificação } \\
\text { Hamada }\end{array}$ & $\begin{array}{c}\text { Classificação } \\
\text { Visotsky }\end{array}$ & $\begin{array}{l}\text { Tratamento } \\
\text { pré-operatório }\end{array}$ & $\begin{array}{c}\text { Lesões } \\
\text { associadas }\end{array}$ & $\begin{array}{l}\text { Tempo de } \\
\text { seguimento } \\
\text { (meses) }\end{array}$ & $\begin{array}{c}\text { Tipo e } \\
\text { tamanho } \\
\text { da prótese }\end{array}$ & $\begin{array}{c}\text { EFO } \\
\text { pré- } \\
\text { operatório }\end{array}$ & $\begin{array}{c}\text { EFO } \\
\text { pós- } \\
\text { operatório }\end{array}$ & UCLA & Neer \\
\hline 1 & 72 & M & + & & 49 & IV & IA & Fisiot e Infilt & $\begin{array}{l}\text { Rotura } \\
\text { bíceps }\end{array}$ & 110 & $\begin{array}{c}\mathrm{ECC} 45 \mathrm{CL} \\
11 \times 125\end{array}$ & (130; 45; L1) & $(150 ; 60 ; T 12)$ & $\begin{array}{l}8+8+5+ \\
3+5=29\end{array}$ & $S$ \\
\hline 2 & 72 & $\mathrm{~F}$ & + & & 42 & IV & IA & Fisiot e Infilt & Nenhuma & 66 & $\begin{array}{c}\text { ECC } 45 \text { CM } \\
\text { Centrada } \\
11 \times 125\end{array}$ & (40; 10; L5) & $(150 ; 60 ;$ L3) & $\begin{array}{c}8+10+5+ \\
3+5=31\end{array}$ & $S$ \\
\hline 3 & 78 & $\mathrm{~F}$ & + & & 96 & IV & IA & Fisiot e Infilt & $\begin{array}{l}\text { Tendinite } \\
\text { biceps }\end{array}$ & 40 & $\begin{array}{c}\text { ECC } 45 \mathrm{CL} \\
11 \times 125\end{array}$ & $(130 ; 0 ;$ L5) & (150; 20; L1) & $\begin{array}{c}10+10+5+ \\
3+5=33\end{array}$ & $S$ \\
\hline 4 & 72 & $F$ & + & + & 49 & IV & $\| B$ & Nenhum & $\begin{array}{c}\text { Deltóide } \\
\text { denervado }\end{array}$ & 26 & $\begin{array}{c}\text { ECC } 45 \mathrm{CL} \\
11 \times 125\end{array}$ & (105; 25; L1) & $(20 ; 0 ; S 1)$ & $\begin{array}{c}10+4+0+ \\
3+5=22\end{array}$ & 1 \\
\hline 7 & 44 & $\mathrm{~F}$ & + & + & 48 & IV & IB & Fisiot e Infilt & Nenhuma & 45 & $\begin{array}{c}\text { ECC } 41 \mathrm{CL} \\
9 \times 150 \\
\text { Deslocada } \\
\text { P1 }\end{array}$ & $(95 ; 35 ;$ L1) & $(100 ; 40 ; \mathrm{T} 12)$ & $\begin{array}{l}6+8+4+ \\
3+5=26\end{array}$ & S \\
\hline 8 & 66 & $\mathrm{~F}$ & + & & 96 & IV & $\| \mathrm{A}$ & Nenhum & $\begin{array}{l}\text { Bíceps auto- } \\
\text { tenodesado }\end{array}$ & 70 & $\begin{array}{c}\text { ECC } 41 \mathrm{CL} \\
7 \times 125 \\
\text { Centrada }\end{array}$ & (105; 25; L1) & $(95 ; 35 ;$ L2) & $\begin{array}{l}4+8+4+ \\
3+5=24\end{array}$ & I \\
\hline 9 & 71 & $F$ & + & & 60 & $\|$ & IA & Fisiot & $\begin{array}{l}\text { Tenodese } \\
\text { biceps }\end{array}$ & 66 & $\begin{array}{c}\text { ECC } 41 \mathrm{CL} \\
9 \times 125 \\
\text { Centrada }\end{array}$ & $(120 ; 50 ; T 10)$ & $(100 ; 40 ;$ L3) & $\begin{array}{l}8+6+3+ \\
3+5=25\end{array}$ & $S$ \\
\hline
\end{tabular}

$\mathrm{M}$ = Masculino; $\mathrm{F}=$ Feminino; Fisiot = Fisioterapia; Infilt = Infiltração; $\mathrm{ECC}=$ Prótese Eccentra; $\mathrm{CL}=$ Colo longo; $\mathrm{CM}=$ Colo médio; $\mathrm{P} 1$ = Primeiro orifício deslocado para posterior; $\mathrm{P} 2$ = Segundo orifício deslocado para posterior; EFO = Exame físico ortopédico com amplitude de movimento; UCLA = University of California Los Angeles - método de avaliação dos resultados pósoperatórios; Neer = Critérios de Neer com objetivos limitados; S = Satisfatório; I = Insatisfatório. 


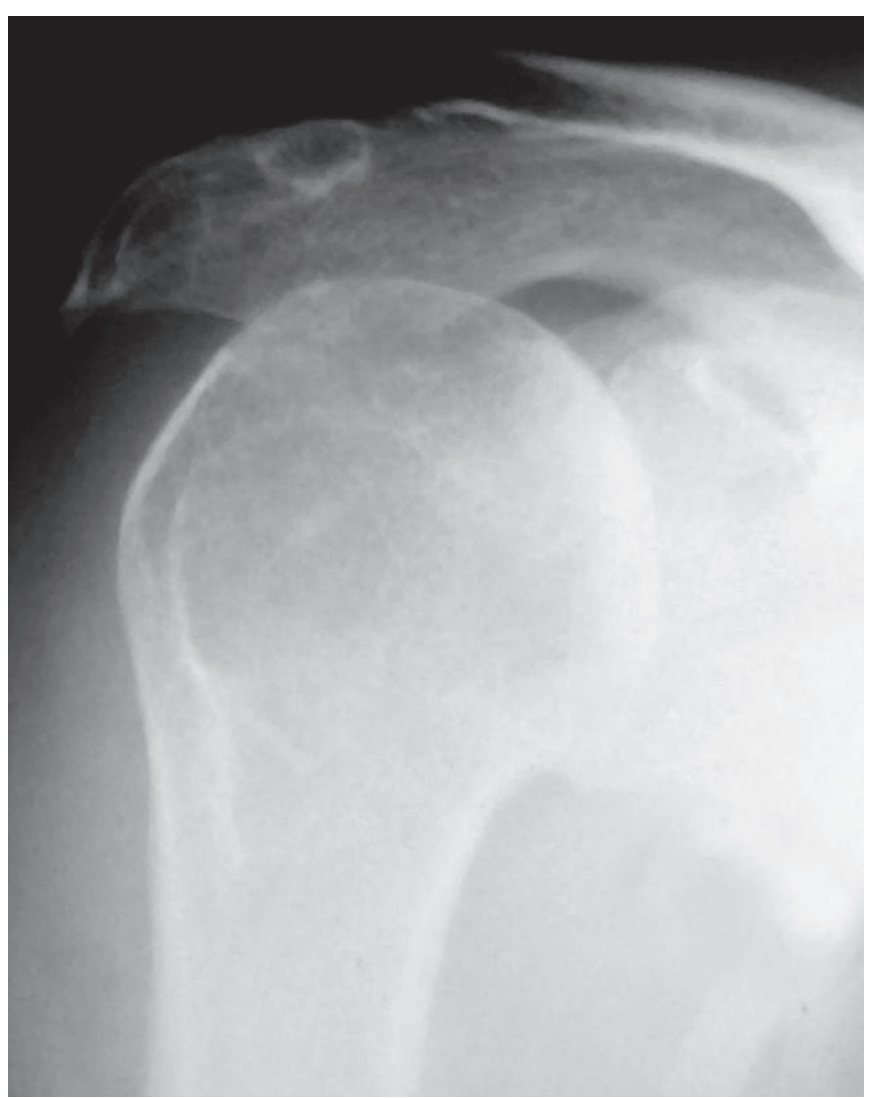

Figura 1 - Imagem radiográfica pré-operatória de ombro direito (caso 1) evidenciando artropatia do ombro por lesão do manguito rotador classificada como Hamada IV e Visotsky IA

Exames radiográficos foram realizados em todos os pacientes seguindo a padronização proposta por Doneux et al, para permitir melhor avaliação pré e pósoperatória $^{(9)}$ (figura 1).

Todos os casos foram classificados com base nos critérios radiográficos propostos por Hamada et $a l^{(3)}$. Estes autores definiram cinco estágios baseados em radiografias, considerando principalmente o intervalo acrômio-umeral (IAU) como indicador sensível de LMR extensa: a distância de 6 a $7 \mathrm{~mm}$ tomada como limite mínimo para ombros normais ${ }^{(3)}$. Classificamos um caso como grau II (IAU inferior ou igual a $6 \mathrm{~mm}$ ); um caso como grau III, pois ocorreu a acetabulização ${ }^{1 *}$ (aumen-

$1 *$ acetabulização é neologismo utilizado por Hamada et al, por analogia com a articulação do quadril onde a cabeça femoral é a responsável pelo desenvolvimento do acetábulo.

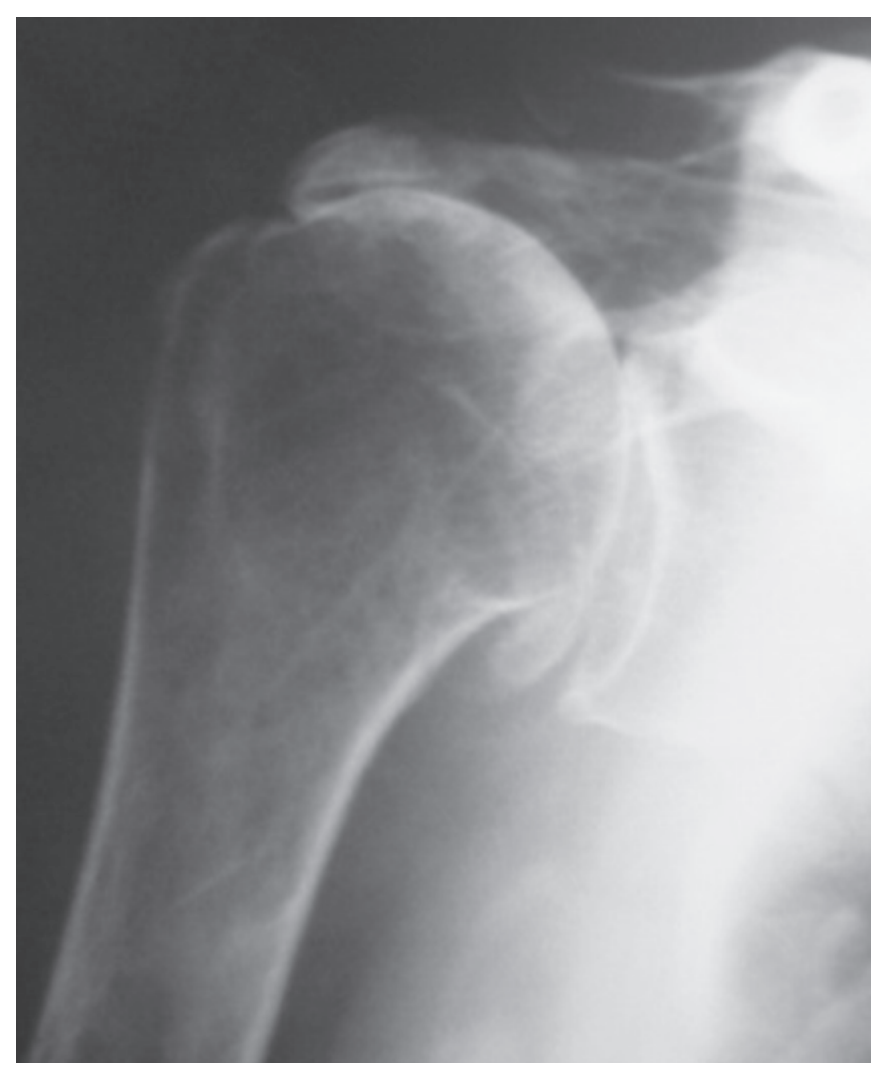

Figura 2 - Imagem radiográfica pré-operatória de ombro direito (caso 11) evidenciando artropatia por lesão do manguito rotador classificada como Hamada IV e Visotsky IIA. Este é um caso em que se observa a acetabulização.

to da concavidade) do acrômio associado aos achados encontrados no grau II; oito casos como grau IV (redução do espaço articular do ombro, associada às alterações encontradas no grau III) (figura 2) e um caso como grau $\mathrm{V}$, porque foi observado o colapso da cabeça do úmero, característico de artropatia do MR (tabela 1).

Utilizamos também os critérios radiográficos propostos por Visotsky et al, que consideram a quantidade de descentralização da articulação do ombro e a migração superior da cabeça do úmero como relacionados, principalmente com a extensão da LMR, a integridade do arco CA e o grau e direção da erosão óssea da cavidade glenoidal ${ }^{(4)}$. Classificamos cinco casos como tipo IA, em que a articulação é considerada centralizada e estável; um caso como tipo IB, em que, apesar de centralizada, a articulação já está medializada devido à erosão medial da glenóide; quatro casos como 

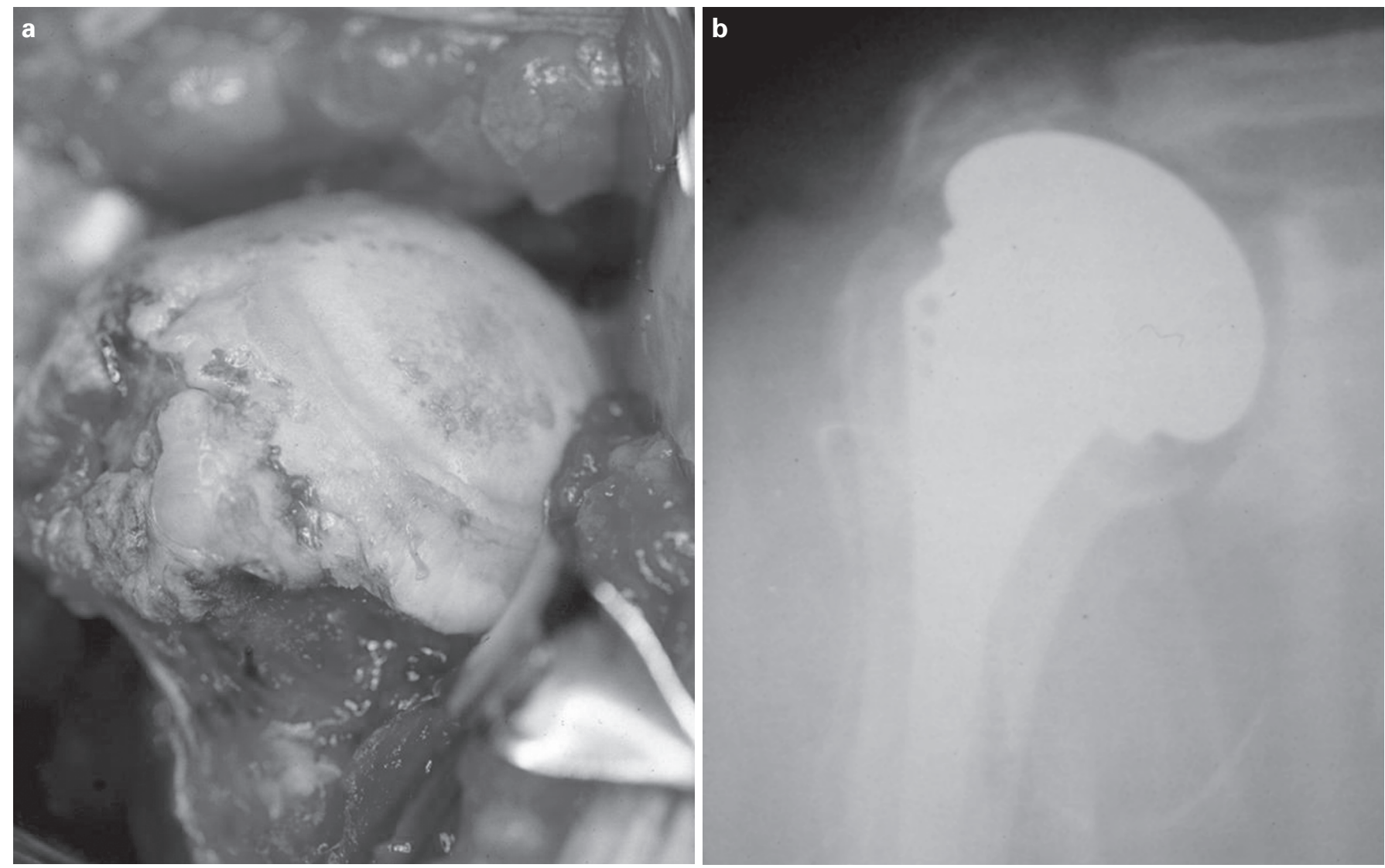

Figura 3 - Caso 1. (a) Imagem intra-operatória de ombro com artrose grave e com ausência dos tendões do manguito rotador, (b) Imagem radiográfica pós-operatória imediata de ombro direito mostrando a prótese Eccentra.

tipo IIA, que seria uma descentralizada com estabilidade limitada, pois o arco CA está muito comprometido, havendo erosão súpero-medial da glenóide; e um caso como tipo IIB, descentralizada e instável, em que os estabilizadores anteriores estão comprometidos e há ausência da estabilização pelo arco CA (tabela 1). Foram feitos exames de imagem de ressonância magnética do ombro acometido durante a avaliação préoperatória de cinco pacientes $(45,4 \%)$. Em todos, assim avaliados, obteve-se a confirmação da LMR extensa e irreparável. Todos os pacientes foram submetidos à artroplastia parcial do ombro pela via de acesso deltopeitoral (figuras $3 \mathrm{a}$ e b). Os modelos de implante utilizados encontram-se na tabela 1. Foram encontradas lesões do tendão da cabeça longa do músculo bíceps braquial (CLB) em seis pacientes (54,6\%), completas (rupturas) em dois pacientes, e parcial em outros quatro. Foi feita a tenodese da CLB em dois pacientes (tabela 1). O tempo de seguimento variou de 27 a 183 meses, com média de 69 meses. Os métodos escolhidos para avaliação dos pacientes no período pós-operatório basearam-se nos critérios da UCLA (University of California at Los Angeles) como descrito por Ellman et $a l^{(10)}$, no índice de satisfação dos pacientes em relação ao alívio da dor; e no método de avaliação de objetivos limitados, proposto por Neer et $a l^{(7)}$. A medida da amplitude articular do ombro foi realizada pelos parâmetros descritos pela American Academy of Orthopaedic Surgeons, como descrito por Hawkins et $a^{(11)}$.

\section{RESULTADOS}

Utilizando o método de avaliação da escala UCLA ${ }^{(10)}$, observamos a média de 22 pontos, sendo o resultado considerado bom em três pacientes, regular em três e ruim em cinco pacientes. $\mathrm{O}$ índice de satisfação dos 


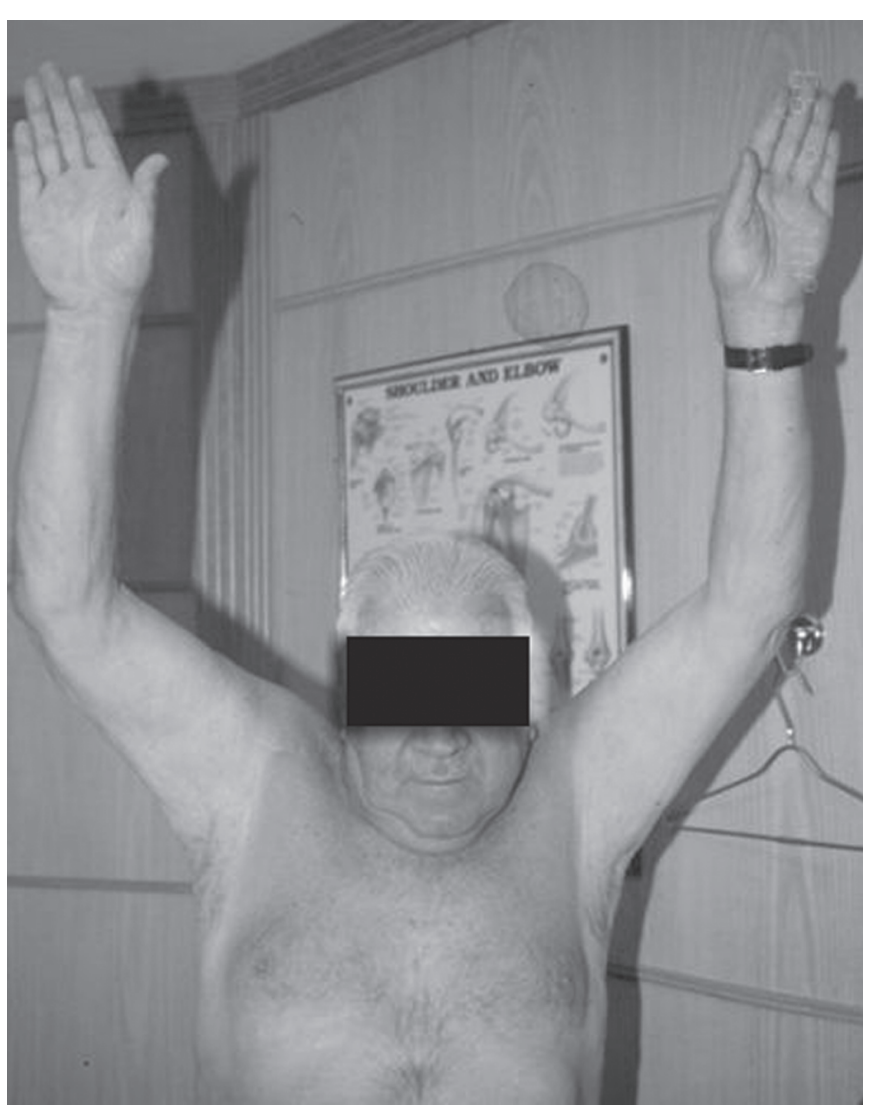

Figura 4 - Imagem de paciente (caso 1) no pós-operatório tardio, classificado como resultado satisfatório

pacientes em relação ao alívio da dor foi de $81,8 \%$ (tabela 1).

Por meio do método de avaliação de objetivos limitados, proposto por Neer et $a^{(7)}$, o resultado foi considerado satisfatório em seis pacientes $(54,6 \%)$ (figura 4) e insatisfatório em cinco (figura 5a; tabela 1).

Com relação à medida da amplitude articular do ombro, identificamos redução da elevação ativa média, variando de $106^{\circ}$ no período pré-operatório, para $93^{\circ}$ no pós-operatório. A rotação lateral média aumentou, variando de $24^{\circ}$ no período pré-operatório, para $35^{\circ}$ no pós-operatório. A média da rotação medial variou do nível da $1^{\mathrm{a}}$ vértebra lombar (L1) no período pré-operatório, para o nível da $2^{\mathrm{a}}$ vértebra lombar (L2) no pós-operatório (tabela 1). Observamos complicações em três pacientes, sendo ascensão da prótese em dois (figura 5b) e soltura da prótese em um.
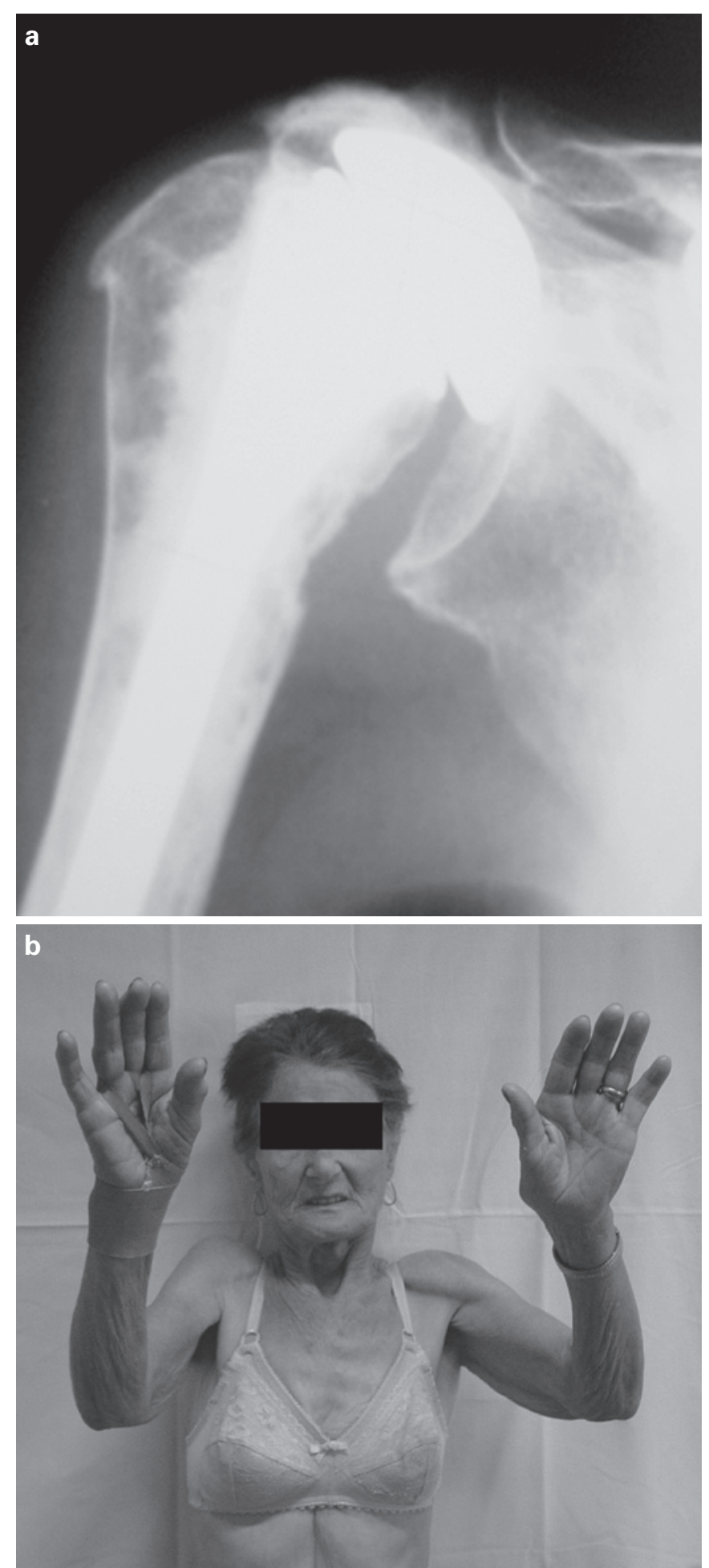

Figura 5 - Caso 11. (a) Imagem radiográfica pós-operatória de ombro direito (caso 11) evidenciando ascensão da prótese que se está articulando com o arco coracoacromial, (b) Imagem da paciente no pós-operatório tardio classificada como resultado insatisfatório. 


\section{DISCUSSÃO}

A artropatia por LMR é desafio para os cirurgiões ortopedistas. Existem várias técnicas para seu manejo, apresentando todas elas resultados pouco previsíveis $^{(2,4,12-14)}$.

Observamos na literatura que a idade de aparecimento da artropatia variou de 50 a 80 anos. Na nossa casuística, a média de idade foi de 69,1 anos, variando de 44 a 78 anos. O sexo feminino e o acometimento do membro dominante foram observados em $90,9 \%$ da nossa casuística, concordando com os dados apre-

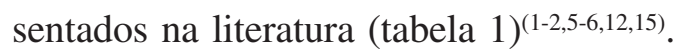

Visotsky et al obtiveram $89 \%$ de resultados satisfatórios com a utilização da artroplastia parcial para o tratamento dos pacientes classificados como tipo IA, IB e IIA, conseguindo alívio da dor e acréscimo na elevação e rotação lateral do ombro. Não incluíram em seu estudo os pacientes classificados como tipo IIB $^{(4)}$.

Em nossa casuística observamos que apenas um caso classificado como tipo IA apresentou resultado insatisfatório e todos os outros pacientes classificados como tipo IA e IB tiveram evolução satisfatória. Apenas um caso classificado como tipo IIA apresentou-se de forma satisfatória; os outros pacientes, classificados como tipo IIA e IIB, tiveram evolução insatisfatória. Observamos que os casos em que os estabilizadores anteriores do ombro permaneceram intactos, com mínima migração superior da cabeça umeral, evoluíram de forma satisfatória. Esse fato nos faz sugerir que a classificação de Visotsky é eficaz e nos possibilita estimar o resultado final da artroplastia, apesar de o pequeno número de casos não nos permitir realizar análise estatística comparativa (tabela 1).

Estudos encontrados na literatura relatam desfechos semelhantes aos observados em nossa casuística, como o de Sanchez-Sotelo et al, que obtiveram $67 \%$ de resultados satisfatórios e amplitude de movimento pósoperatória de $91^{\circ}$ de elevação, $41^{\circ}$ de rotação lateral e rotação medial atingindo o nível da 1 a vértebra lombar $^{(6)}$ e o estudo de Field et al, no qual são relatados resultados satisfatórios em $62,5 \%$ dos casos $^{(12)}$.

Williams et al apontaram o fortalecimento da musculatura do MR remanescente e principalmente da por- ção anterior do músculo deltóide como sendo fator essencial para obtenção da função do membro superior acima da cabeça. Conseguiram resultados superiores aos nossos, com $86 \%$ de resultados satisfatórios pelo método de avaliação de objetivos limitados de Neer, obtendo média na elevação ativa do ombro de $120^{\circ}$ e rotação lateral ativa de $46^{\circ}$ no pós-operatório $^{(15)}$.

Zuckerman et al utilizaram o método de avaliação pós-operatória baseado nos critérios da UCLA, obtendo média de 22 pontos, com amplitude de movimento médio de $86^{\circ}$ de elevação ativa e $29^{\circ}$ de rotação lateral ativa. Relataram $87 \%$ de satisfação dos pacientes com relação ao alívio da dor ${ }^{(2)}$. Estes resultados mostraramse muito semelhantes aos encontrados em nossa casuística, em que obtivemos pontuação média de 22,7 pontos e índice de satisfação de $81,8 \%$ em relação ao alívio da dor (tabela 1).

O resultado favorável da artroplastia parcial no tratamento da artrose do ombro associada à LMR depende principalmente da integridade do arco CA e da musculatura do deltóide intacta e funcional, condições que

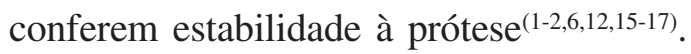

Observamos alguns fatores relacionados à evolução insatisfatória em nossa casuística, como: realização de cirurgia prévia com comprometimento da integridade do arco CA, lesões prévias do músculo deltóide, radiografia evidenciando ascensão da prótese e soltura da prótese (tabela 1). Sanchez-Sotelo et al identificaram a descompressão subacromial prévia e a migração proximal da prótese como fatores relacionados à evolução insatisfatória. Eles não encontraram associação significativa entre o tamanho da prótese umeral utilizada e o alívio da dor, a amplitude de movimento, a instabilidade do ombro e o resultado final ${ }^{(6)}$.

\section{CONCLUSÃO}

A artroplastia parcial de ombro para o tratamento da artropatia por lesão do manguito rotador promove alívio do quadro doloroso e permite alguma melhora na função do membro para realização das atividades diárias; entretanto, é uma técnica que leva a altos índices de resultados insatisfatórios. 


\section{REFERÊNCIAS}

1. Pollock RG, Deliz ED, McIlveen SJ, Flatow EL, Bigliani LU. Prosthetic replacement in rotator cuff-deficient shoulders. J Shoulder Elbow Surg. 1992;1(4):173-86.

2. Zuckerman JD, Scott AJ, Gallagher MA. Hemiarthroplasty for cuff tear arthropathy. J Shoulder Elbow Surg. 2000;9(3):16972.

3. Hamada K, Fukuda H, Mikasa M, Kobayashi Y. Roentgenographic findings in massive rotator cuff tears. A long-term observation. Clin Orthop Relat Res. 1990;(254):926.

4. Visotsky JL, Basamania C, Seebauer L, Rockwood CA, Jensen KL. Cuff tear arthropathy: pathogenesis, classification, and algorithm for treatment. J Bone Joint Surg Am. 2004;86-A Suppl 2:35-40.

5. Neer CS 2nd, Craig EV, Fukuda H. Cuff-tear arthropathy. J Bone Joint Surg Am. 1983;65(9):1232-44.

6. Sanchez-Sotelo J, Cofield RH, Rowland CM. Shoulder hemiarthroplasty for glenohumeral arthritis associated with severe rotator cuff deficiency. J Bone Joint Surg Am. 2001;83A(12):1814-22.

7. Neer CS 2nd, Watson KC, Stanton FJ. Recent experience in total shoulder replacement. J Bone Joint Surg Am. 1982;64(3): 319-37.

8. Franklin JL, Barrett WP, Jackins WP, Matsen FA 3rd. Glenoid loosening in total shoulder arthroplasty. Association with rotator cuff deficiency. J Arthroplasty. 1988;3(1):39-46.

9. Doneux PS, Checchia SL, Miyazaki AN. Padronização do estudo radiográfico da cintura escapular. Rev Bras Ortop. 1998;33(11):883-8.
10. Ellman H, Hanker G, Bayer M. Repair of the rotator cuff. Endresult study of factors influencing reconstruction. J Bone Joint Surg Am. 1986;68(8):1136-44.

11. Hawkins RJ, Bokor DJ. Clinical evaluation of shoulder problems. In: Rockwood CA Jr, Matsen FA 3rd, editors. The Shoulder. 2nd ed. Saint Louis: W.B. Saunders; 1998. p. 164-98.

12. Field LD, Dines DM, Zabinski SJ, Warren RF. Hemiarthroplasty of the shoulder for rotator cuff arthropathy. J Shoulder Elbow Surg. 1997;6(1):18-23.

13. Sirveaux F, Favard L, Oudet D, Huquet D, Walch G, Molé D. Grammont inverted total shoulder arthroplasty in the treatment of glenohumeral osteoarthritis with massive rupture of the cuff. Results of a multicentre study of 80 shoulders. J Bone Joint Surg Br. 2004;86(3):389-95.

14. Guery J, Favard L, Sirveaux F, Oudet D, Mole D, Walch G. Reverse total shoulder arthroplasty. Survivorship analysis of eighty replacements followed for five to ten years. J Bone Joint Surg Am. 2006;88(8):1742-7.

15. Williams GR, Rockwood CA Jr. Hemiarthroplasty in rotator cuff-deficient shoulders. J Shoulder Elbow Surg. 1996;5(5): 362-7.

16. Arntz CT, Matsen FA 3rd, Jackins S. Surgical management of complex irreparable rotator cuff deficiency. J Arthroplasty. 1991;6(4):363-70.

17. Arntz CT, Jackins S, Matsen FA 3rd. Prosthetic replacement of the shoulder for the treatment of defects in the rotator cuff and the surface of the glenohumeral joint. J Bone Joint Surg Am. 1993;75(4):485-91. Erratum in: J Bone Joint Surg Am. 1993; 75(7):1112. 